\title{
BMJ Open In-hospital myocardial infarction and adherence to evidence-based drug therapies: a real-world evaluation
}

\author{
Salvatore Soldati (D , , Mirko Di Martino (D) , ${ }^{1}$ Davide Castagno, ${ }^{2}$ Marina Davoli, ${ }^{1}$ \\ Danilo Fusco ${ }^{1}$
}

To cite: Soldati S, Di Martino M, Castagno D, et al. In-hospital myocardial infarction and adherence to evidence-based drug therapies: a real-world evaluation. BMJ Open 2021;11:e042878. doi:10.1136/ bmjopen-2020-042878

- Prepublication history for this paper is available online. To view these files, please visit the journal online (http://dx.doi org/10.1136/bmjopen-2020042878).

Received 20 July 2020 Revised 08 December 2020 Accepted 21 January 2021

Check for updates

(C) Author(s) (or their employer(s)) 2021. Re-use permitted under CC BY-NC. No commercial re-use. See rights and permissions. Published by BMJ.

${ }^{1}$ Department of Epidemiology, Lazio Regional Health Service, Rome, Italy

${ }^{2}$ Division of Cardiology, Department of Medical Sciences, University of Turin, Turin, Italy

Correspondence to

Dr Mirko Di Martino; m.dimartino@deplazio.it

\section{ABSTRACT}

Objectives This study aimed to measure adherence to chronic polytherapy following an acute myocardial infarction (AMI) and to find out associations between adherence and the setting of AMI onset (in vs out of hospital) as well as other determinants.

Design Retrospective follow-up study.

Setting Population living in the Lazio Region, Italy. Participants This study included 25779 hospitalised patients with a first diagnosis of AMI in 2012-2016, after the exclusion of those with hospital admission for AMI or related causes in the previous 5 years.

Primary and secondary outcome measures Patients were classified as in-hospital AMI (IH-AMI) or out of hospital AMI (OH-AMI) according to present-onadmission codes. Adherence was measured based on prescription claims during a 6-month follow-up after hospital discharge, using medication possession ratio (MPR). Adherence to chronic polytherapy was defined as MPR $\geq 75 \%$ to at least 3 of the following medications: antithrombotics, betablockers, ACE inhibitors/angiotensin receptor blockers and statins.

Results Among the entire cohort, 1044 (4\%) patients suffered IH-AMI. Overall, 15440 (60\%) patients were deemed adherent to chronic polytherapy. Female gender, older age, mental disorders, renal disease, asthma and ongoing concomitant treatments were factors associated with poor adherence. By contrast, patients with more severe AMI and those already taking evidence-based (E-B) drugs were more likely to be adherent. A strong association between the setting of AMI onset and adherence was observed: IH-AMI patients were $46 \%$ less likely to be adherent to E-B medications during their 6-month follow-up as compared with $\mathrm{OH}-\mathrm{AMI}$ patients (OR $0.54 ; 95 \% \mathrm{Cl} 0.47$ to $0.62 ; \mathrm{p}<0.001$ ).

Conclusion Pharmacotherapy is not consistent with clinical guidelines, especially for IH-AMI patients. Our findings provide evidence on a previously unidentified groups of patients at risk for poor adherence, who might benefit from greater medical attention and dedicated healthcare interventions.

\section{INTRODUCTION}

Most studies investigating acute myocardial infarction (AMI) epidemiology have target patients with AMI admitted via the community emergency medical system or through

\section{Strengths and limitations of this study}

The population-based design, many patients involved and the integration of health information systems to define and analyse the patient's care pathway.

- This is the first study evaluating the adherence to chronic polytherapy post acute myocardial infarction (AMI), taking into account, the setting of AMI onset (in vs out of hospital).

- This study uses multilevel modelling techniques to control for any variability on medication adherence attributable to hospitals of discharge.

- Misclassification of drug utilisation may have occurred because the dosage instructions were not known, and the defined daily doses were used as the dosage assumption.

- Although all available potential confounders were considered to adjust for differences in patients' characteristics, the possibility of unmeasured confounding remains.

the emergency department (out of hospital AMI (OH-AMI)). Findings from these observational studies have informed risk factors and optimal treatment of AMI, contributing to a progressive reduction in overall mortality and risk of recurrent AMI worldwide. ${ }^{12}$ It is increasingly recognised, however, that there are patients whose symptoms onset of AMI begin after being hospitalised for other medical conditions. ${ }^{34}$ Little is known, in literature, about patients experiencing in-hospital AMI (IH-AMI). One such recent study focused on the incidence, risk factors and mortality outcomes related to IH-AMI. ${ }^{5}$ Regardless of the setting of onset of AMI, evidence-based (E-B) secondary prevention strategies are based on changes in lifestyle and E-B drug therapy. With this regard, international guidelines recommend the combined use of drugs belonging to different anatomical therapeutic chemical (ATC) groups including antithrombotic agents, $\beta$-blockers, 
ACE inhibitors (ACEIs), angiotensin receptor blockers (ARBs) and statins. ${ }^{67}$

Poor medication adherence after AMI is a worldspread problem, which compromises patient outcomes and increases patient mortality. Post-AMI survival benefit deriving from long-term adherence to guidelines recommended polytherapy has been clearly shown in literature. $^{8-14}$ However, observational studies highlighted suboptimal use and poor compliance in the general post-AMI population and in specific subset of affected individuals. ${ }^{11} 1415$

Moreover, the transition of care from hospital to the community-based setting might also represents an important aspect to be taken into account when assessing medication adherence: patients discharged from a specialised hospital ward (eg, cardiology, cardiac surgery, coronary care units) were found to be associated with higher adherence rates. ${ }^{14-16-18}$ Typically, the hospital takes care of patients in the 'first phase' of follow-up period. After this period, patients are definitively managed by cardiologists in the community-based setting. However, different hospitals have different follow-up protocols, according to the length of follow-up period and frequency of evaluation. These differences in healthcare delivery generate heterogeneity in the population and raise equity issues in terms of quality and effectiveness of the transition care from the acute setting to the outpatient setting. For these reasons, our research hypothesis is that the setting in which AMI develops may significantly impact on the probability of being discharge by specialised hospital wards and, consequently, on the recommended therapeutic strategies and adherence to them.

Therefore, the main objectives of this study were: (1) to measure, in a real-world scenario, the adherence to chronic polytherapy following an AMI; (2) to identify determinants of adherence to E-B drugs specifically focusing on the potential association between setting of onset of AMI (ie, IH-AMI vs OH-AMI).

\section{METHODS}

\section{Data sources}

Our Department has access to health information systems of the Lazio region of Italy that contain mortality, hospital admission and drug claims data. We collected data from the Regional Hospital Information Systems (HIS), the Regional Admission and Discharge Information System (RAD), the Regional Healthcare Emergency Information System (HEIS), the Mortality Information System (MIS) and the Regional Drug Dispense Registry.

The HIS is an integrated information system designed to collect clinical and administrative information regarding hospital admissions for each patient discharged from public and private hospitals of the Lazio region. The HIS includes patients' characteristics (single anonymous identifier, gender, date and place of birth, and place of residence); admission and discharge dates; discharge diagnoses (up to 6); procedure codes (up to 6) according to the International Classification of Disease, Ninth Revision, Clinical Modification (ICD-9-CM); hospital admission and discharge ward and a regional code that corresponds to the admitting facility.

Since July 2008, tracking of additional information about hospital discharge record has been activated in the Lazio region thanks to RAD Information System (corporate decision nr. D4118). The ministerial directive of December 2010 establishes 'the integration of the HIS with additional mandatory sections for the collection of additional information about hospital discharge data'. RAD collects additional information on comorbidities (eg, time to surgery, the presence of AMI diagnosis code at hospital admission time). This information is useful to characterise the severity of patient's condition at the time of hospitalisation or surgery. These additional data are inserted into the RAD forms at the time of patient's hospital discharge, when the diagnostic and therapeutic care pathways are clearly defined.

The HEIS includes all visits occurred in emergency departments of the Lazio region and collects patient demographic characteristics, admission information, visit and discharge dates and hours, ICD-9-CM diagnosis at discharge, reported symptoms on arrival, status at discharge (eg, dead, hospitalised or discharged at home) and triage score.

Information on drugs reimbursed by the national healthcare system and dispensed by public and private pharmacies or by hospital pharmacies at discharge is available from the Regional Drug Dispense Registry. The data available on each prescription include patient's identification number, prescribing physician's number, ATC code of the drug purchased, number of packs, number of units per pack, dosage, unit cost per pack and prescription date.

Any date of death was obtained from the MIS.

Data from different information systems have been integrated using a deterministic record linkage procedure based on unique and anonymous subject identifier. In this way, we created a chronological, demographical, residential, clinical and healthcare-related patient profile.

\section{Setting and study cohort}

The present observational study was based on the population living in the Lazio region, Italy. Using data from the regional HIS, the study included a cohort of all patients discharged from hospitals between 1 January 2012 and 31 December 2016 with a diagnosis of AMI. AMI was defined according to ICD-9-CM codes 410.xx (first or second diagnosis position). In case of multiple hospital admissions, the first admission during the study period was defined as the index admission. Subsequent hospitalisations for any reason were recorded, and repeated admissions within 2 days of discharge were regarded as one single 'episode of care'.

Classification as to whether AMI occurred in-hospital was based on present-on-admission codes from RAD Information System, which provides information regarding 
diagnostic codes (present, absent, presence cannot be deduced from clinical documentation, not applicable) at the time of hospital presentation. AMI patients with admission code diagnosis (present) were classified as OH-AMI, patients without admission code diagnosis (absent) were classified as IH-AMI. Admission code diagnosis (present or absent) was available in more than $98 \%$ of AMI patients. To improve identification of unambiguously IH-onset AMI, we excluded patients with unclear admission code diagnosis ('presence cannot be deduced from clinical documentation' or 'not applicable'). In such manner, we should be able to reduce the possible misclassification of exposure due to critical situations, in which patients may have ambiguous diagnosis at the time of hospital admission.

Patients aged 18-100 years at discharge were screened for inclusion in the study. Only incident cases of AMI were included: patients with hospital admission for AMI or related causes (ie, percutaneous coronary intervention, bypass or surgery of the heart and great vessels) in the 5 years before index admission were excluded.

Patients who were not registered in the regional health assistance file at time of discharge from hospital were excluded (note that healthcare assistance in Italy is offered to all resident citizens without restrictions). Finally, patients who had an individual follow-up shorter than 30 days were excluded, to give all patients the chance to achieve clinical stability and to guarantee a minimum observation period of 1 month for consistently estimate adherence to polytherapy.

\section{Patient and public involvement \\ No patient involved.}

\section{Patient characteristics}

Patients were characterised according to sociodemographic factors (age, gender), comorbidities that might contraindicate prescription of specific ATC group drugs, previous use of E-B drugs, previous use of other (nonE-B) medications, previous hospitalisation with a diagnosis of mental disorders (ICD-9-CM codes: 290-319), hospital discharge ward and ST-elevation myocardial infarction (STEMI) as indicator of severity of disease. STEMI patients were identified using ICD-9-CM diagnosis codes 410.xx, excluding 410.7x (non-ST-elevation AMI) and 410.9x (acute AMI, not otherwise specified) in any diagnostic position. The following diseases were assessed by health ticket exemption or during hospitalisation or emergency department visit for index admission as well as in the 2 years preceding the beginning of follow-up: asthma (ICD-9-CM diagnosis code 493), renal disease (ICD-9-CM diagnosis codes: 582-588, V.42.0, V.45.1, V.56, ICD-9-CM procedure codes: 38.95, 39.95, 54.98, 55.6), sinoatrial bradycardia (ICD-9-CM diagnosis code 427.8). These clinical conditions might contraindicate drug prescription of specific ATC groups due to potential adverse effects (eg, $\beta$-blockers in patients suffering from asthma).
We used the number of distinct, non-E-B drugs, prescribed in the 6 months preceding the beginning of follow-up as a crude measure of ongoing concomitant treatments. Medications with the same first five digits of the ATC code were considered as a group. ${ }^{19}$

Moreover, to better define patients' clinical profile, during the 6 months preceding follow-up initiation, information was also collected on the use of all E-B drugs: antithrombotic agents, $\beta$-blockers, ACEIs, ARBs and statins.

\section{Follow-up}

We evaluated medication use 'immediately' after the acute event, by analysing prescription patterns during the 6 months following discharge from the index admission. Follow-up started the same date of hospital discharge of the index episode of AMI. The end of follow-up coincided either with the end of 6-month follow-up, the date of death or with the date of all-cause hospitalisation whichever came first. The last 'censoring' criterion allows one to measure the net impact of the hospital that has discharged the patient on medication adherence without the potential interference of subsequent hospitalisations.

\section{Definition of exposure and outcome}

AMIs were classified as IH-AMI or OH-AMI according to 'present-on-admission' codes retrieved using the RAD Information System which provides information regarding diagnostic codes (present or absent) at the time of hospital presentation.

The main outcome of the study was adherence to chronic poly-therapy at 6-month follow-up. All drugs in this study were included in the patients' healthcare plans and were equally available to all residents, in accordance with the universal healthcare coverage provided to residents of Italy. Information about prescriptions of antithrombotics (ATC: B01AC06, B01AC04, B01AC05, B01AC22, B01AC24, B01AF01, B01AF02, B01AF03, B01AA03, B01AA07, B01AE07), $\beta$-blockers (ATC: C07), ACEI/ARBs (ATC: C09) and statins (ATC: C10AA) were retrieved for all patients. Adherence to medication was measured through the medication possession ratio (MPR), calculated as the number of days of medication supplied during the follow-up on the basis of defined daily doses (DDDs) divided by the number of calendar days in the follow-up. Adherence to individual medications was defined as an MPR $\geq 0.75$. Adherence to chronic polytherapy was defined as a MPR $\geq 0.75$ for at least three of the four E-B drugs. ${ }^{13} 14$

\section{Statistical analysis}

Data are presented as frequencies and percentages for categorical variables and mean value $\pm \mathrm{SD}$ for continuous variables. Considering the hierarchical data structure (patients are nested within hospitals), logistic multilevel models were performed to take into account potential intraclass correlation. The variance components were expressed in terms of median odds ratio (MOR), a measure that quantifies the variability between clusters, in this 
case between different hospitals of discharge. The MOR quantifies the variation between clusters by comparing two persons from two randomly chosen different clusters. Consider two persons with the same covariates, chosen randomly from two different clusters. MOR is the median odds ratio between the person of higher propensity and the person of lower propensity. This measure is always greater than or equal to 1 . MOR equal to 1 indicates no variability between clusters, as the variability between group increases MOR value increases. ${ }^{20}$ In a first step, MOR was estimated using an intercept-only model. In a second step, MOR was estimated controlling for patient characteristics, to ensure that of the heterogeneity of patients within groups (in terms of age, comorbidities or severity of AMI) did not influence the estimates of variance.

Logistic multilevel models were also applied to identify determinants of adherence to E-B drugs, considering the correlation within clusters. Determinants of adherence were selected based on a priori knowledge ${ }^{21}{ }^{22}$ : gender and age, discharge ward, ST-elevation AMI, use of E-B drugs (ie, antithrombotics, $\beta$-blockers, ACEI/ ARBs, statins) during the 6 months prior to the index admission (defined as at least one prescription), ongoing concomitant treatments (ie, number of distinct non-E-B drugs) and relevant comorbidities retrieved from the hospital records for both the index admission and the two previous years.

Results were expressed as OR, 95\% CI and $\mathrm{p}$ values. Statistical analyses were carried out using Stata software, V.15 (StataCorp.2015: Release 15).

\section{RESULTS}

\section{The study cohort}

The flow chart in figure 1 shows the selection process of the study cohort. Of the 34854 patients discharged from hospital with a first diagnosis of AMI between 1 January 2012 and 31 December 2016, 25779 (74\%) met the

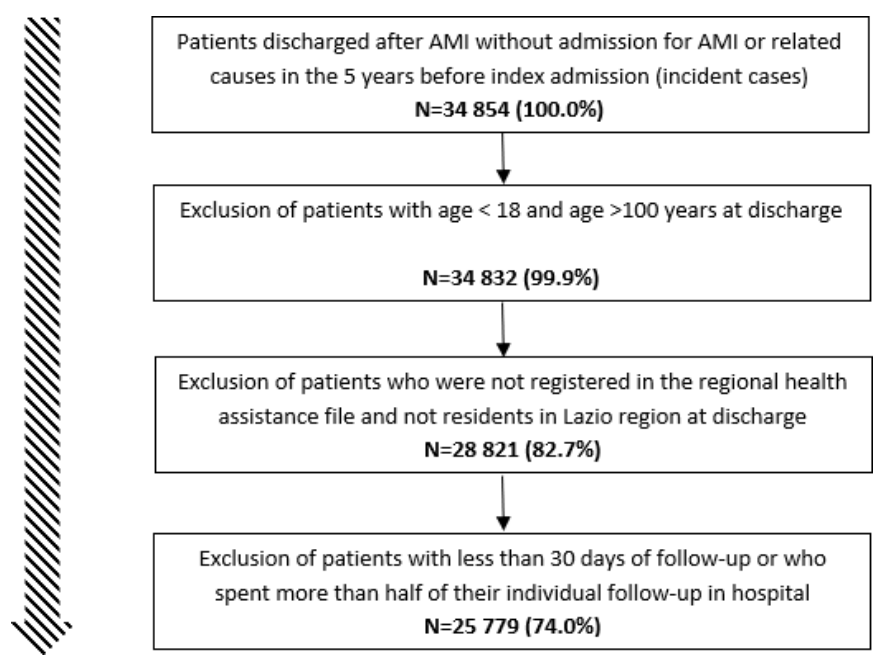

Figure 1 Cohort selection. Exclusion criteria flow chart. AMI, acute myocardial infarction. inclusion criteria and were enrolled in the present study. Mean age was 68 years, $17138(66 \%)$ were male (table 1$)$. Overall, $11108(43 \%)$ of patients suffered an AMI with ST segment elevation and the largest number of patients $20207(78 \%)$ was discharged from cardiology wards. More than $65 \%$ of patients had at least a prescription of E-B medications ( $\beta$-blockers, antithrombotics, ACEI/ ARBs or statins) during the 6 months prior to the index admission. Overall, more than two-thirds of patients were receiving concomitant treatments at the time of AMI and the prevalence of these treatments showed a parallel increase with age.

Among the entire cohort, 1044 (4.0\%) patients suffered an IH-AMI. They were older, had more comorbidities (eg, renal disease, asthma and mental disorders) and less frequently had a diagnosis of ST-elevation AMI (31\% vs $44 \%$ ) compared with patients experiencing an OH-AMI. In addition, the use of at least one E-B medication before hospitalisation was greater among patients suffering an IH-AMI compared with OH-AMI (78\% vs $66 \%)$. Patients suffering IH-AMI also showed a higher prevalence of ongoing concomitant treatments (number of distinct non-E-B drugs prescribed in the 6 months preceding the beginning of follow-up) and less likely were discharged from cardiology wards (48\% vs $80 \%$ ).

\section{Post-AMI adherence to E-B medications}

The adherence to E-B medications by gender and age group is reported in table 2. Statins were characterised by the highest adherence (78\%), followed by antithrombotics (69\%), ACEI/ARBs (63\%) and $\beta$-blockers (50\%). Lower adherence was observed among women, most notably for statins and antithrombotics $(14 \%$ and $12 \%$ points lower than men, respectively). This gender difference was attenuated as age increased. Older age groups showed lower adherence to all medications. The adherence to each of the recommended drugs decreased markedly, for both males and females, moving from the age group ' $75-84$ ' to the group ' 85 +' years.

Overall, 15440 (60\%) patients were adherent to chronic polytherapy (as per protocol definition) following an AMI. However, only 6463 (25\%) patients were adherent to the full combination of E-B treatments considered in this study. Women were less likely to be treated with a combination of E-B drugs compared with males $(51 \%$ vs $64 \%)$. This gender difference was less pronounced as age increased (table 3 ).

A strong variability in adherence to chronic polytherapy between different hospitals of discharge was observed, even after controlling for patients' characteristics. As reported in tables 4 and 5 and a higher and statistically significant ( $p=$ value: 0.042 ) variability among discharge hospitals was observed for patients suffering IH-AMI (MOR 1.57; 95\% CI 1.33 to 2.06; $\mathrm{p}=0.019$ ) as compared with OH-AMI (MOR 1.46; $95 \%$ CI 1.33 to 1.64 ; $\mathrm{p}<0.001$ ).

Using logistic multilevel model, determinants of adherence to chronic polytherapy were determined (table 6). A lower probability of adherence was observed 
Table 1 Baseline characteristics of the study cohort

\begin{tabular}{|c|c|c|c|}
\hline & $\begin{array}{l}\text { Total cohort } \\
25.779(100 \%)\end{array}$ & $\begin{array}{l}\text { IH-AMI } \\
1.044(4.0 \%)\end{array}$ & $\begin{array}{l}\text { OH-AMI } \\
24.735(96.0 \%)\end{array}$ \\
\hline & $\mathbf{N}(\%)$ & $\mathrm{N}(\%)$ & $\mathbf{N}(\%)$ \\
\hline \multicolumn{4}{|l|}{ Age group (years) } \\
\hline $18-54$ & $4702(18.24)$ & $101(9.67)$ & 4601 (18.6) \\
\hline $55-64$ & $5886(22.83)$ & $149(14.27)$ & $5737(23.19)$ \\
\hline $65-74$ & $6387(24.78)$ & $243(23.28)$ & $6144(24.84)$ \\
\hline $75-84$ & $6122(23.75)$ & $360(34.48)$ & $5762(23.29)$ \\
\hline $85+$ & $2682(10.4)$ & $191(18.3)$ & $2491(10.07)$ \\
\hline Age, mean (SD), years & $67.61(13.20)$ & 73.19 (12.52) & $67.37(13.18)$ \\
\hline Gender (men) & $17138(66.48)$ & $590(56.51)$ & $16548(66.9)$ \\
\hline ST-elevation AMI & $11108(43.09)$ & $319(30.56)$ & $10789(43.62)$ \\
\hline Renal disease & $2335(9.06)$ & $166(15.9)$ & $2169(8.77)$ \\
\hline Sinoatrial bradycardia & $249(0.97)$ & $10(0.96)$ & $239(0.97)$ \\
\hline Asthma & $188(0.73)$ & $12(1.15)$ & $176(0.71)$ \\
\hline Mental disorders & $1098(4.26)$ & $97(9.29)$ & $1001(4.05)$ \\
\hline \multicolumn{4}{|l|}{$\begin{array}{l}\text { Ongoing concomitant treatments (distinct } \\
\text { group of drugs) }\end{array}$} \\
\hline $0-1$ & $7587(29.43)$ & $180(17.24)$ & 7407 (29.95) \\
\hline $2-4$ & $8507(33)$ & $293(28.07)$ & $8214(33.21)$ \\
\hline $5-7$ & $5236(20.31)$ & $272(26.05)$ & $4964(20.07)$ \\
\hline $8-10$ & $2688(10.43)$ & $161(15.42)$ & $2527(10.22)$ \\
\hline$>10$ & $1761(6.83)$ & $138(13.22)$ & $1623(6.56)$ \\
\hline E-B drugs use (at least one prescription) ${ }^{*}$ & $17083(66.27)$ & $811(77.68)$ & $16272(65.79)$ \\
\hline Discharge ward (cardiology) & 20207 (78.39) & 501 (47.99) & 19706 (79.67) \\
\hline
\end{tabular}

*Prescribed in the 6 months preceding the index admission.

AMI, acute myocardial infarction; E-B, evidence based; IH-AMI, in-hospital acute myocardial infarction; OH-AMI, out of hospital acute myocardial infarction.

in women (OR $0.75 ; 95 \%$ CI 0.71 to $0.79 ; \mathrm{p}<0.001)$ and elderly patients. With this regard, the effect of age was not completely linear: with respect to the reference category (age less than 55 years): the probability of adherence increased in the age group ' $55-64$ ' years (OR 1.12; $95 \%$ CI 1.03 to 1.22; $p=$ value: 0.007 ) but decreased, although not significantly, in the group ' $65-74$ ' years (OR 0.98 ; 95\% CI 0.90 to $1.07 ; \mathrm{p}=0.618$ ). A significant drop in the probability of adherence was observed in older age groups ('75-84' years OR $0.67 ; 95 \%$ CI 0.61 to $0.73 ; \mathrm{p}<0.001, \geq 85$ years; OR $0.40 ; 95 \%$ CI 0.35 to $0.44 ; \mathrm{p}<0.001$ ). A similar trend was observed for the ongoing concomitant treatments in the 6 months before index admission.

In addition, lower adherence to chronic polytherapy was observed among patients with comorbidities. In contrast, a significantly higher adherence to poly-therapy was observed among patients already taking E-B drugs in the 6 months prior index admission (OR $1.57 ; 95 \%$ CI 1.47 to $1.67 ; \mathrm{p}<0.001)$ and among patients suffering from an ST-elevation AMI (OR 1.48; 95\% CI 1.40 to 1.56 ; $\mathrm{p}<0.001$ ). Finally, a lower probability of adherence was observed in patients discharged from unspecialised hospital wards as compared with those who discharged from cardiology ward (OR $0.58 ; 95 \%$ CI 0.54 to $0.63 ; \mathrm{p}<0.001$ ).

After adjustment for potential confounders (including age, gender, renal disease, sinoatrial bradycardia, asthma, mental disorders, ST-elevation AMI, ongoing concomitant treatments and E-B drugs use during the 6 months prior to hospitalisation) patients suffering IH-AMI were $46 \%$ less likely to be adherent to polytherapy as compared with OH-AMI patients (OR 0.54; 95\% CI 0.47 to 0.62 ; $\mathrm{p}<0.001$ ). As summarised in table 7, IH-AMI patients showed significantly lower adherence levels for three of four E-B drugs, that is, statins, antithrombotics and ACEI/ARBs. This 'gap' was less significant for beta-blockers.

\section{DISCUSSION}

\section{Prevalence and clinical characteristics of IH-AMI patients}

AMI occurring in patients who have already been admitted to the hospital for other clinical conditions is an entity that has been poorly investigated so far. In this study, among all the patients experiencing AMI between 1 January 2012 and 31 December 2016 in Lazio region (see 
Table 2 Adherence to evidence-based medications by gender and age group

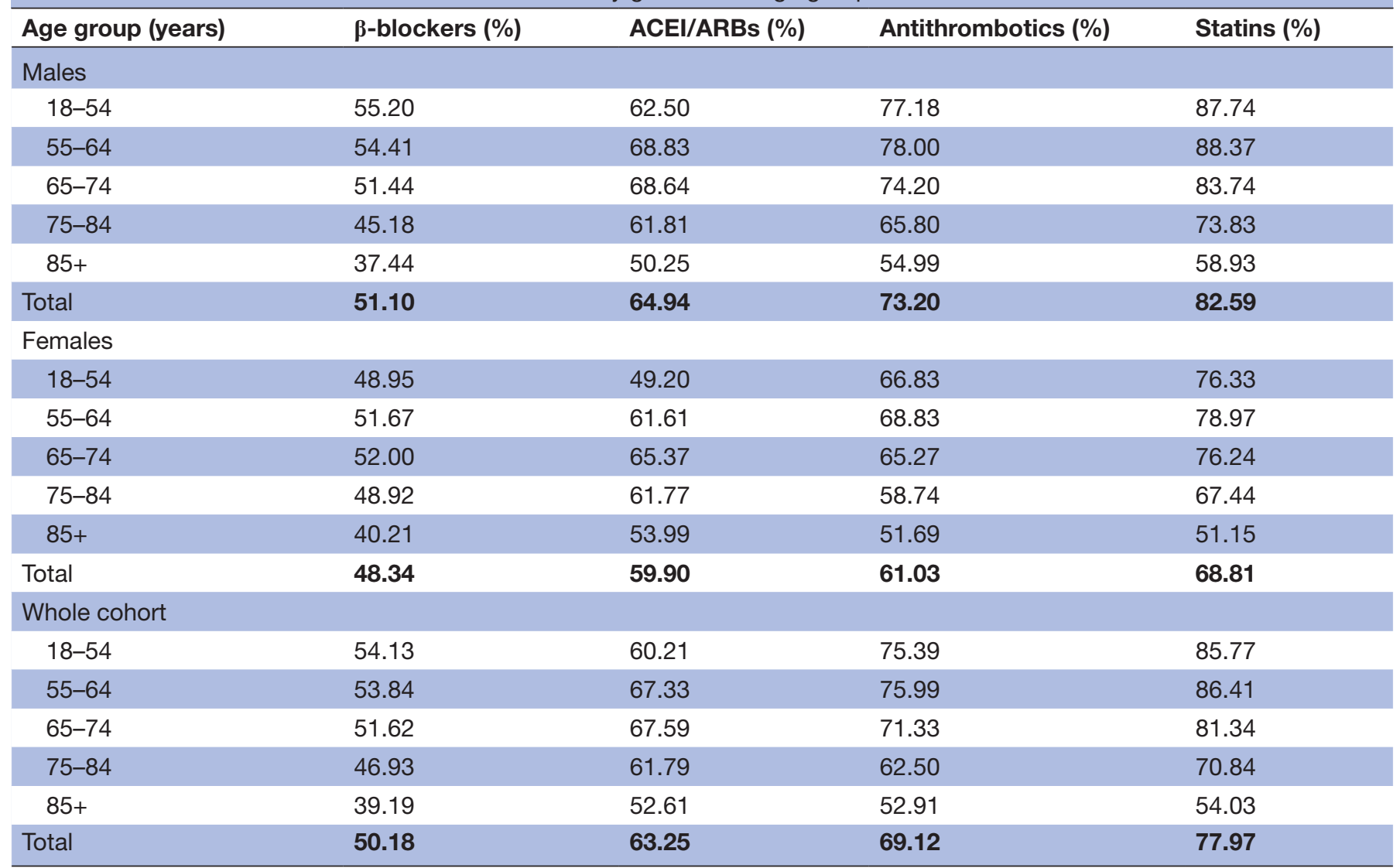

ACEI/ARBs, ACE inhibitors/angiotensin receptor blockers.

cohort selection in figure 1), the proportion of patients with IH-AMI of all patients with AMI was $4.0 \%$. Our study has several key findings. First, compared with OH-AMI patients, those suffering IH-AMI were more often female, older, and less likely to be discharged from cardiology wards, possibly reflecting a higher burden of comorbidities. Indeed, IH-AMI patients had more often a history of renal disease, asthma, mental disorders and more frequently were treated with beta-blockers, antithrombotic agents, ACE-Is/ARBs or statins in the 6 months prior the index event Interestingly, IH-AMI patients less frequently suffered from ST-elevation AMI. Much of these findings are concordant with the observations from a previous study by Zahn et $a l .^{23}$ In addition, Maynard $e t$ $a \hat{l}$ reported that patients who had AMI while hospitalised for other medical conditions were older, more likely to have atypical symptoms, and had higher rates of renal disease, cerebrovascular disease, congestive heart failure, diabetes mellitus, chronic obstructive pulmonary disease, dementia and cancer than patients who presented as OH-AMI to the Department of Veterans Affairs Health System.

Second, and possibly even more important, we observed that patients experiencing IH-AMI were less likely to be adherent to E-B medications for secondary prevention of AMI during 6-month follow-up. Moreover IH-AMI patients were more likely to be discharged from non-cardiological wards and this may have negatively impacted on the quality of care after the acute event.

\section{Adherence to chronic polytherapy}

Concerning the whole study period, we found that after a hospital discharge for AMI, only $60 \%$ of patients were deemed adherent to polytherapy in the following 6 months. Treatments with proven benefit in secondary prevention following AMI were underused in this study. This result is alarming if we consider that our definition of adherence was not very restrictive (ie, adherence defined as MPR $\geq 75 \%$ for at least three of the four predefined E-B drugs) and that adherence was evaluated only for the first 6 months after AMI (adherence should be greater in the initial stages of care and may decrease over time) ${ }^{24}$ Our findings are consistent with the results of other investigations, which reported unsatisfactory prescribing rates of E-B therapies after AMI during different time frames ${ }^{15}$ and in different countries. ${ }^{21} 2225$

To the best of our knowledge, our study was the first to assess, whether adherence differed between patients who had IH-AMI as compared with those who experienced OH-AMI. Interestingly, the setting of AMI onset had a significant impact on polytherapy adherence. In fact, patients who had AMI during their hospital stay were less likely to be adherent to chronic polytherapy compared with patients who had AMI outside of the hospital. In 
Table 3 Adherence to chronic polytherapy by gender and age group

\begin{tabular}{|c|c|c|}
\hline $\begin{array}{l}\text { Age group } \\
\text { (years) }\end{array}$ & $\begin{array}{l}\text { Adherence (\%) (MPR } \\
\geq 75 \% \text { at least } 3 \text { of } 4 \\
\text { E-B drugs) }\end{array}$ & $\begin{array}{l}\text { Adherence (\%) } \\
\text { (MPR } \geq 75 \% \text { for all } \\
4 \text { E-B drugs) }\end{array}$ \\
\hline \multicolumn{3}{|l|}{ Males } \\
\hline 18-54 & 67.95 & 32.20 \\
\hline $55-64$ & 70.53 & 32.47 \\
\hline $65-74$ & 67.12 & 27.72 \\
\hline $75-84$ & 54.05 & 20.12 \\
\hline $85+$ & 39.15 & 11.81 \\
\hline Total & 64.13 & 27.66 \\
\hline \multicolumn{3}{|l|}{ Females } \\
\hline $18-54$ & 51.91 & 23.55 \\
\hline $55-64$ & 60.64 & 25.67 \\
\hline $65-74$ & 58.88 & 24.59 \\
\hline $75-84$ & 51.08 & 18.06 \\
\hline $85+$ & 36.37 & 11.53 \\
\hline Total & 51.49 & 19.93 \\
\hline \multicolumn{3}{|c|}{ Whole cohort } \\
\hline $18-54$ & 65.19 & 30.71 \\
\hline 55-64 & 68.47 & 31.06 \\
\hline 65-74 & 64.47 & 26.71 \\
\hline $75-84$ & 52.66 & 19.16 \\
\hline $85+$ & 37.40 & 11.63 \\
\hline Total & 59.89 & 25.07 \\
\hline
\end{tabular}

E-B, evidence based; MPR, medication possession ratio.

crude logistic multilevel model, IH-AMI patients were $53 \%$ less likely to be adherent as compared with OH-AMI patients (OR 0.47; 95\% CI 0.41 to $0.54 ; \mathrm{p}<0.001$ ). After adjustment for potential confounders, this relationship was only slightly attenuated but remained strongly significant (OR 0.54; $95 \%$ CI 0.47 to 0.62; $\mathrm{p}<0.001$ ). Moreover, we observed a greater variability in terms of adherence to multiple recommended secondary prevention therapies for IH-AMI patients. This finding might reflect the lack of standardised and homogeneous clinical care pathways within hospitals of discharge for patients who have suffered AMI during hospitalisation for other medical conditions. Of note, estimates were adjusted for all variables identified as determinants of adherence to polytherapy such as age, gender, renal disease, sinoatrial bradycardia, asthma, mental disorders, ST-elevation AMI, ongoing concomitant treatments and E-B drugs use during the 6 months prior to hospitalisation. Although being discharge from a specialised hospital ward (eg, cardiology, cardiac surgery, coronary care units) was found to be associated with higher adherence rates, we decided not to adjust for discharge ward because we felt it could be a proxy for setting of AMI onset. IH-AMI patients were less likely discharged from cardiology wards (48\% vs $80 \%$ ) and this reflects a different care pathway for those compared with patients who had OH-AMI. In this situation, an adjustment for discharge ward, could have introduced (rather than eliminated) a bias (overadjustment). ${ }^{26}$

We also found that female gender, older age, mental disorders, renal disease, asthma and ongoing concomitant treatments were significantly associated with nonadherence to chronic polytherapy. Conversely, adherence was positively and significantly associated with patients who had a severe form of disease (ST-elevation AMI) and patients who have already begun E-B drugs in the 6 months before index admission.

Our findings are consistent with the results of other investigations. It is notable that the current study demonstrates that women are receiving less optimal medical therapy in all age groups and all drug categories. The clinical relevance of gender differences varies by age and type of medication. For example, small differences are observed in the use of beta-blockers, larger differences are observed in the use of statins. Smolina $e t a l^{27}$ confirmed these gender differences and showed that treatment was less often initiated in women. Older age was also found to be associated with lower adherence in several previous studies. ${ }^{151718}$ A higher prevalence of cognitive disorders, memory impairment and limited ability to absorb new information in the elderly population have been associated with lower adherence. ${ }^{28}$ Tuppin $e t a l^{18}$ reported that adherence to E-B treatment was decreased significantly by an age greater than 74 years, confirming our findings. The prescription of complex regimens including multiple drugs has been widely acknowledged as a barrier to patient adherence ${ }^{29}$ : the longer the list of drugs prescribed, the lower the adherence of patients. Chronic conditions like asthma, sinoatrial bradycardia and renal disease reduce drug prescription of specific ATC groups due to adverse effects and contraindications increasing the probability of poor adherence to chronic polytherapy. A previous hospitalisation with a diagnosis of mental disorders decreased the odds of adherence: the mechanisms by which mental disorders can affect adherence may include poor motivation, pessimism about treatment

Table 4 Variation between clusters for OH-AMI patients: the MORs

\begin{tabular}{lllll}
\hline Multilevel model & Level of analysis & Explanatory variables & MOR (95\% CI) & P value \\
\hline Two-level regression & (Patients) - HoD & Intercept only & $1.71(1.50$ to 2.02$)$ & $<0.001$ \\
Two-level regression & (Patients) - HoD & Patient's characteristics & $1.46(1.33$ to 1.64$)$ & $<0.001$ \\
\hline
\end{tabular}

$\mathrm{HoD}$, hospital of discharge; MOR, median OR; OH-AMI, out of hospital acute myocardial infarction. 
Table 5 Variation between clusters for IH-AMI patients: the MORs

\begin{tabular}{lllll}
\hline Multilevel model & Level of analysis & Explanatory variables & MOR (95\% Cl) & P value \\
\hline Two-level regression & (Patients) - HoD & Intercept only & $1.69(1.43$ to 2.16$)$ & 0.005 \\
Two-level regression & (Patients) - HoD & Patient's characteristics & $1.57(1.33$ to 2.06)
\end{tabular}

AMI, acute myocardial infarction; HoD, hospital of discharge; IH-AMI, in-hospital acute myocardial infarction; MOR, median OR.

effectiveness, diminished attention, memory and cognition, decreased self-care and even intentional self-harm. ${ }^{30}$ Moreover, patients suffering from ST-elevation AMI or those who had already begun E-B drugs before index AMI were more likely to be adherent to chronic polytherapy. The former have had a more severe form of the disease and were probably more carefully monitored and made aware of the long-term benefits generated by a continuous and persistent drug treatment. The latter were already used to the chronic and continuous intake of those drugs that are recommended for the secondary prevention of AMI, as a sort of 'inertial effect'.

Table 6 Association between adherence to chronic polytherapy and symptom onset (IH-AMI vs OH-AMI), sociodemographics and clinical characteristics

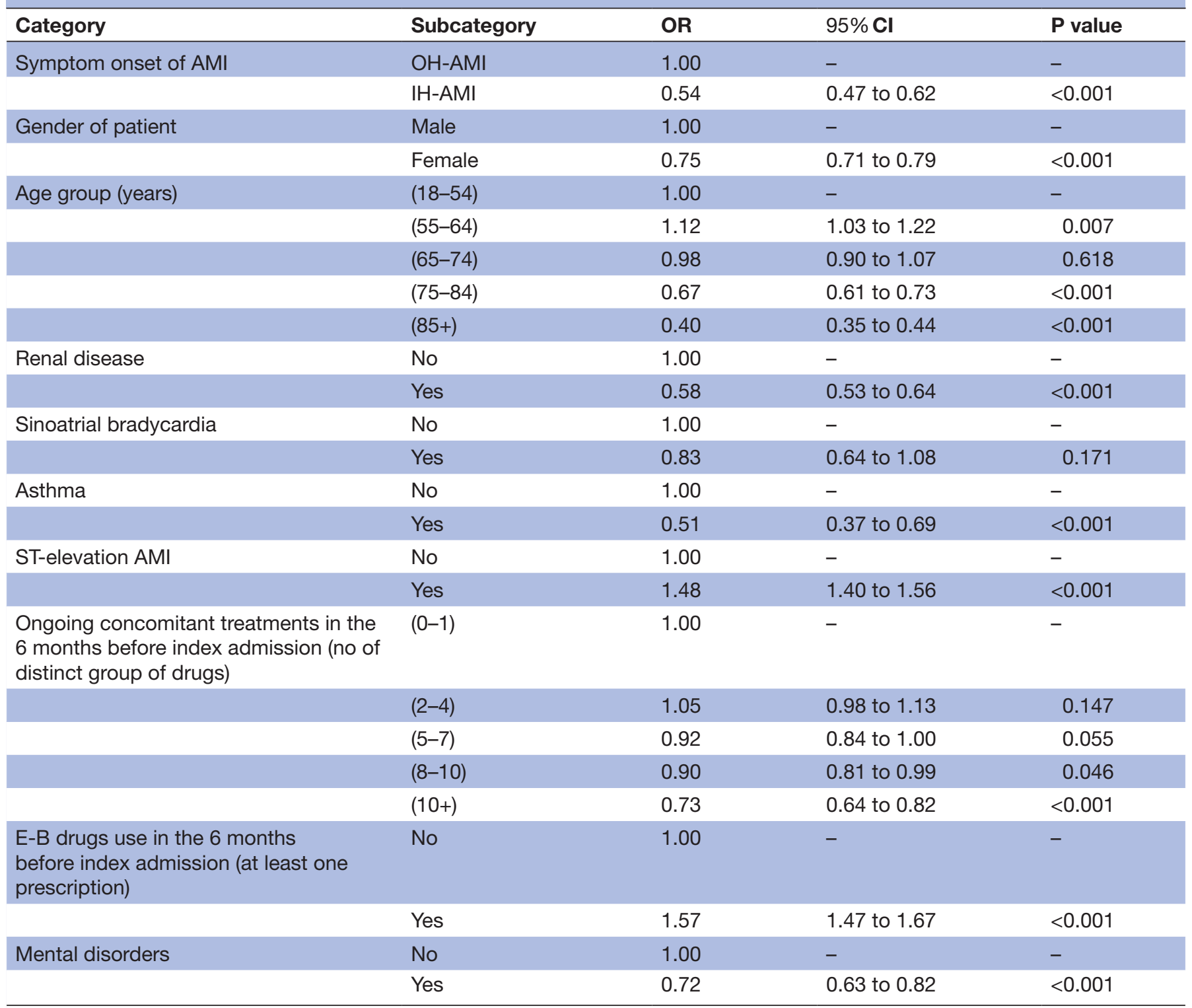

AMI, acute myocardial infarction; E-B, evidence based; IH-AMI, in-hospital acute myocardial infarction; OH-AMI, out of hospital acute myocardial infarction. 
Table 7 Adherence to evidence-based medications by the setting of AMI onset

\begin{tabular}{lllll}
\hline Symptom onset of AMI & $\boldsymbol{\beta}$-blockers (\%) & ACEI/ARBs (\%) & Antithrombotics (\%) & Statins (\%) \\
\hline OH-AMI & 50.24 & 63.85 & 69.88 & 78.78 \\
IH-AMI & 48.66 & 48.95 & 51.15 & 58.72 \\
Whole cohort & 50.18 & 63.25 & 69.12 & 77.97 \\
\hline
\end{tabular}

ACEI/ARBs, ACE inhibitors/angiotensin receptor blockers; IH-AMI, in-hospital acute myocardial infarction; OH-AMI, out of hospital acute myocardial infarction.

\section{Strengths and limitations of the study}

The population-based design, many patients involved and the opportunity to integrate many sources of data to define and analyse the patient's care pathway are the main strengths of this study. Moreover, to our knowledge, this is the first study to evaluate the adherence to E-B medications, considering the setting of AMI onset.

However, the results come from a single region in Italy and may not be generalisable to the other Italian regions due to possible differences in the organisation of regional healthcare services. This notwithstanding, our results are in line with results of similar studies carried out in Italy. ${ }^{31}$ Moreover, our pharmaceutical database does not contain information on the prescribed daily doses and adherence to drug treatment was estimated on the basis of the DDDs. ${ }^{32}$ Using DDDs to calculate drug coverage, we run the risk of not accounting for the real-life dosing of a drug when it is used for other than its principal indication. ${ }^{33}$ Therefore, misclassification of drug utilisation may have occurred because the dosage instructions were not known, and the DDDs were used as the dosage assumption. However, in our study, we tried to overcome this limitation by considering DDDs of beta-blockers reviewed by a panel of physicians, seeing that in secondary prevention post AMI, DDDs are prescribed at lower dosages than the main therapeutic indication.

In addition, MPR method does not depend on whether patients take their medication as prescribed but depends on the prescription given by physicians. Although we cannot be sure that patients actually took the drug, collecting their medications from the pharmacy is a reasonable indication of an intention to continue with therapy: nevertheless, the results of adherence based on claims data may be overestimated.

Finally, although all available potential confounders were included in the models to adjust for differences in patients' characteristics, we cannot exclude that the lack of more detailed clinical data might have caused unmeasured confounding. We tried to counteract this limit by applying several restrictions to obtain a cohort with patients that were as homogeneous as possible.

\section{CONCLUSIONS}

The availability of information systems offers the opportunity to monitor the quality of care and identify weaknesses in public healthcare systems. Although most attention has been paid to patients with AMI admitted via the community emergency medical system or through the emergency department, AMI occurring during hospitalisation for other medical problems is an important clinical problem.

The results of our study show that, in clinical practice, pharmacotherapy for secondary prevention of AMI is not fully consistent with recommended clinical guidelines, especially for IH-AMI patients. Moreover, a strong association between the setting of AMI onset and adherence to multiple E-B drugs was observed. Our findings provide evidence on a previously unidentified groups of patients at risk for poor adherence, who might benefit from greater medical attention and dedicated healthcare interventions. The data strongly support the need for continued efforts to improve adherence to chronic polytherapy post AMI. These findings could also stimulate efforts to implement hospital strategies to give the same 'attention' to IH-AMI patients as OH-AMI patients. In light of the impressive and highly significant impact of the type of discharge ward on the adherence to chronic polytherapy, it is feasible that much of the 'disadvantage' of IH-AMI patients is attributable to the discharge processes, in particular through how far they support effective transitions in and continuity of care. A range of policy tools could be appropriate to reduce this gap, for example, by planning differentiated healthcare transition interventions according to the setting of AMI onset. However, further studies are needed to confirm this association.

Contributors SS contributed to the concept and design of the study, the acquisition of data from the Lazio regional health information systems, the analysis of data and the statistical methodology required for the analytic modelling, the interpretation of results, and the writing of the article. MDM contributed to the design of the study, the statistical methodology required for the analytic modelling, the interpretation of results, and the writing of the article. DC contributed to the clinical interpretation of results, and the writing of the article. MD and DF contributed to the design of the study, and the critical revision of the paper for important intellectual content, and they have given their final approval of the version submitted for publication. All authors agreed to be accountable for all aspects of the work and ensure that questions related to the accuracy or integrity of any part of the work were appropriately investigated and resolved.

Funding The authors have not declared a specific grant for this research from any funding agency in the public, commercial or not-for-profit sectors.

Competing interests None declared.

Patient and public involvement Patients and/or the public were not involved in the design, or conduct, or reporting, or dissemination plans of this research.

Patient consent for publication Not required.

Ethics approval Comitato Etico Lazio 1 (OSS-R-192) Registro Studi Osservazionali.

Provenance and peer review Not commissioned; externally peer reviewed. 
Data availability statement № data are available.

Open access This is an open access article distributed in accordance with the Creative Commons Attribution Non Commercial (CC BY-NC 4.0) license, which permits others to distribute, remix, adapt, build upon this work non-commercially, and license their derivative works on different terms, provided the original work is properly cited, appropriate credit is given, any changes made indicated, and the use is non-commercial. See: http://creativecommons.org/licenses/by-nc/4.0/.

\section{ORCID iDs}

Salvatore Soldati http://orcid.org/0000-0002-1856-4862

Mirko Di Martino http://orcid.org/0000-0002-6033-7591

\section{REFERENCES}

1 Peterson ED, Shah BR, Parsons L, et al. Trends in quality of care for patients with acute myocardial infarction in the National Registry of myocardial infarction from 1990 to 2006. Am Heart $J$ 2008;156:1045-55.

2 Yeh RW, Sidney S, Chandra M, et al. Population trends in the incidence and outcomes of acute myocardial infarction. $N$ Engl $J$ Med 2010;362:2155-65.

3 Maynard C, Lowy E, Rumsfeld J, et al. The prevalence and outcomes of in-hospital acute myocardial infarction in the Department of Veterans Affairs health system. Arch Intern Med 2006;166:1410-6.

4 Erne $\mathrm{P}$, Bertel O, Urban $\mathrm{P}$, et al. Inpatient versus outpatient onsets of acute myocardial infarction. Eur J Intern Med 2015;26:414-9.

5 Bradley SM, Borgerding JA, Wood GB, et al. Incidence, risk factors, and outcomes associated with in-hospital acute myocardial infarction. JAMA Netw Open 2019;2:e187348.

6 National Institute for Clinical Excellence. Secondary prevention in primary and secondary care for patients following a myocardial infarction. NICE guidelines [CG172]. Published date 2013.

7 Smith SC, Benjamin EJ, Bonow RO, et al. AHA/ACCF secondary prevention and risk reduction therapy for patients with coronary and other atherosclerotic vascular disease: 2011 update: a guideline from the American heart association and American College of cardiology Foundation endorsed by the world heart Federation and the preventive cardiovascular nurses association. J Am Coll Cardiol 2011;58:2432-46.

8 Choudhry NK, Glynn RJ, Avorn J, et al. Untangling the relationship between medication adherence and post-myocardial infarction outcomes: medication adherence and clinical outcomes. Am Heart J 2014;167:51-8. e5.

9 Mathews R, Wang W, Kaltenbach LA, et al. Hospital variation in adherence rates to secondary prevention medications and the implications on quality. Circulation 2018;137:2128-38.

10 Hamood H, Hamood R, Green MS, et al. Effect of adherence to evidence-based therapy after acute myocardial infarction on allcause mortality. Pharmacoepidemiol Drug Saf 2015;24:1093-104.

11 Gislason GH, Rasmussen JN, Abildstrøm SZ, et al. Long-term compliance with beta-blockers, angiotensin-converting enzyme inhibitors, and statins after acute myocardial infarction. Eur Heart $J$ 2006;27:1153-8.

12 Korhonen MJ, Robinson JG, Annis IE, et al. Adherence tradeoff to multiple preventive therapies and all-cause mortality after acute myocardial infarction. J Am Coll Cardiol 2017;70:1543-54

13 Kirchmayer U, Di Martino M, Agabiti N, et al. Effect of evidencebased drug therapy on long-term outcomes in patients discharged after myocardial infarction: a nested case-control study in Italy. Pharmacoepidemiol Drug Saf 2013;22:649-57.

14 Di Martino M, Alagna M, Cappai G, et al. Adherence to evidencebased drug therapies after myocardial infarction: is geographic variation related to hospital of discharge or primary care providers? A cross-classified multilevel design. BMJ Open 2016;6:e010926.

15 Kirchmayer U, Agabiti N, Belleudi V, et al. Socio-demographic differences in adherence to evidence-based drug therapy after hospital discharge from acute myocardial infarction: a population-based cohort study in Rome, Italy. J Clin Pharm Ther 2012;37:37-44.

16 Simpson E, Beck C, Richard H, et al. Drug prescriptions after acute myocardial infarction: dosage, compliance, and persistence. Am Heart J 2003;145:438-44.

17 Austin PC, Tu JV, Ko DT, et al. Use of evidence-based therapies after discharge among elderly patients with acute myocardial infarction. CMAJ 2008;179:895-900.

18 Tuppin P, Neumann A, Danchin N, et al. Combined secondary prevention after hospitalization for myocardial infarction in France: analysis from a large administrative database. Arch Cardiovasc Dis 2009;102:279-92.

19 Schneeweiss S, Seeger JD, Maclure M, et al. Performance of comorbidity scores to control for confounding in epidemiologic studies using claims data. Am J Epidemiol 2001;154:854-64.

20 Larsen K, Merlo J. Appropriate assessment of neighborhood effects on individual health: integrating random and fixed effects in multilevel logistic regression. Am J Epidemiol 2005;161:81-8.

21 Ho PM, Bryson CL, Rumsfeld JS. Medication adherence: its importance in cardiovascular outcomes. Circulation 2009;119:3028-35

22 Mathews R, Wang TY, Honeycutt E, et al. Persistence with secondary prevention medications after acute myocardial infarction: insights from the TRANSLATE-ACS study. Am Heart J 2015;170:62-9.

23 Zahn R, Schiele R, Seidl K, et al. Acute myocardial infarction occurring in versus out of the hospital: patient characteristics and clinical outcome. J Am Coll Cardiol 2000;35:1820-6.

24 Di Martino M, Kirchmayer U, Agabiti N, et al. The impact of timewindow bias on the assessment of the long-term effect of medication adherence: the case of secondary prevention after myocardial infarction. BMJ Open 2015;5:e007866.

25 Sanfélix-Gimeno G, Peiró S, Ferreros I, et al. Adherence to evidencebased therapies after acute coronary syndrome: a retrospective population-based cohort study linking Hospital, outpatient, and pharmacy health information systems in Valencia, Spain. J Manag Care Pharm 2013;19:247-57.

26 VanderWeele TJ. On the relative nature of overadjustment and unnecessary adjustment. Epidemiology 2009;20:496-9.

27 Smolina K, Ball L, Humphries KH, et al. Sex disparities in postacute myocardial infarction pharmacologic treatment initiation and adherence: problem for young women. Circ Cardiovasc Qual Outcomes 2015;8:586-92.

28 Sattler ELP, Lee JS, Perri M. Medication (re)fill adherence measures derived from pharmacy claims data in older Americans: a review of the literature. Drugs Aging 2013;30:383-99.

29 Vik SA, Maxwell CJ, Hogan DB. Measurement, correlates, and health outcomes of medication adherence among seniors. Ann Pharmacother 2004;38:303-12.

30 Wang PS, Bohn RL, Knight E, et al. Noncompliance with antihypertensive medications: the impact of depressive symptoms and psychosocial factors. J Gen Intern Med 2002;17:504-11.

31 Filippi A, D'Ambrosio G, Giustini SE, et al. Pharmacological treatment after acute myocardial infarction from 2001 to 2006: a survey in Italian primary care. J Cardiovasc Med 2009;10:714-8

32 World Health Organization,. Guidelines for ATC classification and DDD assignment 2020. Oslo, Norway: Collaborating Centre for Drug Statistics Methodology, 2019.

33 Grimmsmann T, Himmel W. Discrepancies between prescribed and defined daily doses: a matter of patients or drug classes? Eur J Clin Pharmacol 2011;67:847-54. 